\title{
An electrochemical study in aqueous solutions on the binding of dopamine to a sulfonated cyclodextrin host
}

\author{
Gillian M. Hendy ${ }^{*, 1,2}$, Carmel B. Breslin ${ }^{1}$ \\ Department of Chemistry, National University of Ireland Maynooth, Maynooth, Co. Kildare, Ireland
}

\section{A R T I C L E I N F O}

\section{Article history:}

Received 5 July 2011

Received in revised form 20 October 2011

Accepted 22 October 2011

Available online 28 October 2011

\section{Keywords:}

Inclusion complex

$\beta$-Cyclodextrin

Dopamine

Association constant

\begin{abstract}
A B S T R A C T
Clear evidence for the formation of a weak inclusion complex between dopamine (DA) and a sulfonated $\beta-C D$ host in aqueous solution was obtained using a combination of electrochemical approaches. Using cyclic voltammetry, a distinct increase in the oxidation potential of DA and a reduction in the peak oxidation current were observed on adding an excess concentration of the sulfonated $\beta$-CD to the electrolyte solution. Equally, a clear increase in the half-wave oxidation potential of DA was observed in the presence of the sulfonated $\beta-C D$ using rotating disc voltammetry. The association constant, $K$, was computed as $331.3 \pm 5.8$, indicating the formation of a weak inclusion complex, while a 1:1 stoichiometry for the inclusion complex was deduced from a Job's plot analysis. The rate constant for the oxidation of DA was found to decrease on formation of the inclusion complex. This was attributed to higher reorganization energy for the oxidation of the included DA. These changes in the electrochemistry of DA were not observed when an excess of the smaller sulfonated $\alpha-C D$ was added to the electrolyte, indicating that these variations are not connected with simple electrostatic interactions between the protonated DA and the anionic sulfonated groups. It is proposed that the aromatic ring of the DA molecule includes within the cyclodextrin cavity, while the protonated amine group remains outside the cavity, bound electrostatically with the anionic sulfonated groups.
\end{abstract}

(c) 2011 Elsevier Ltd. All rights reserved.

\section{Introduction}

Cyclodextrins (CD) are macrocyclic oligosaccharides composed of $\alpha$-D-glucopyranoside units linked by $\alpha-(1,4)$ bonds. They are generally made up of glucopyranoside units of ${ }^{4} C_{1}$ chair conformation. The three common members are $\alpha-, \beta$ - and $\gamma$-CD, which have 6 , 7 and 8 repeating glucopyranoside units, respectively. These units are orientated in a cyclic manner giving the typical conical or truncated cone structure with a relatively hydrophobic interior and hydrophilic exterior [1]. This structural property gives cyclodextrins the ability to include appropriately sized guests, through non-covalent interactions, such as hydrogen bonding, hydrophobic interactions and electrostatic interactions [2-7].

Cyclodextrins can also be chemically modified to replace the hydroxyl groups on both the primary and secondary rims of the CDs, with a variety of appropriate alkyl or sulfate groups [8]. In

\footnotetext{
* Corresponding author. Tel.: +1617 253 3638; fax: +1617 2588827.

E-mail addresses: ghendy@mit.edu (G.M. Hendy), carmel.breslin@nuim.ie (C.B. Breslin).

1 ISE member.

2 Present address: Koch Institute at MIT, 76-661, 77 Massachusetts Avenue, Cambridge, MA 02139, USA.
}

particular, negatively charged cyclodextrins can be obtained by substitution of either the primary or secondary hydroxyl group of $\beta-C D$ with a sulfonate group [9]. On the primary rim there are seven potential substitution sites corresponding to the C-6 positions, while, on the secondary rim there are 14, represented by the C-2 and C-3 positions, as shown in Fig. 1. Amini et al. [10] reported that substitution of these CDs is predominantly at the C-2 and C-6 positions, while, Chen et al. [11] confirmed nearly complete sulfation at the C- 6 position of the primary hydroxyl groups and partial sulfation at the C-2 secondary hydroxyl groups. They also reported no substitution at the $\mathrm{C}-3$ positions. From these reports it can be concluded that almost the entire primary rim and some of the secondary rim is sulfonated and negatively charged. Sulfonated $\beta$-CDs are used in chromatography and especially in capillary electrophoresis for the enantiomeric separation of compounds [12]. However, there have been very few studies carried out on the inclusion of guest molecules within the cavity of the sulfonated $\beta$-CDs.

In this paper we report on the formation of an inclusion complex between sulfonated $\beta-C D$ and dopamine (DA). DA was chosen as it represents a large family of amine-based drugs. DA is an interesting guest as it is protonated at near-netural $\mathrm{pH}$, providing a positively charged guest molecule. In addition, dopamine is electroactive, being readily oxidized into the o-dopaminoquinone [13]. This means that the formation of the inclusion complex can 


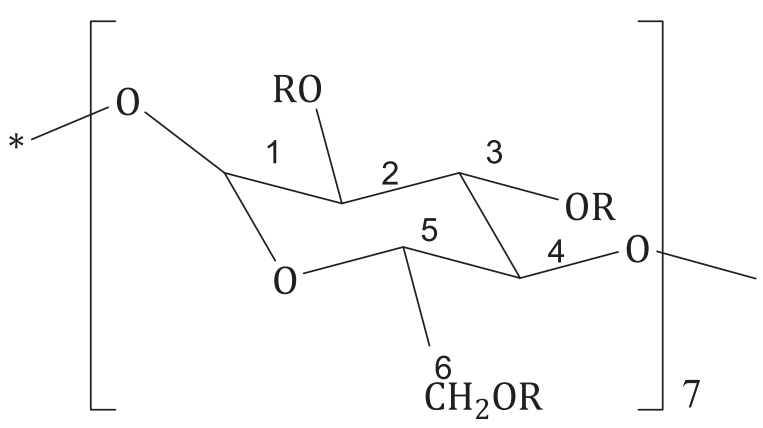

Fig. 1. Chemical Structure of $\beta-C D$, with the $C$ atoms numbered from 1 to 6 .

be studied using electrochemical techniques. This electrochemical approach is used frequently to study the formation of an inclusion complex [14-18]. For example, Strelets et al. [19] have studied the formation of an inclusion complex between ferrocene and $\beta-C D$ using cyclic voltammetry by monitoring the oxidation of ferrocene in the absence and presence of the neutral $\beta-C D$. They explained the positive shift in the oxidation potential and the reduction in the peak oxidation current for ferrocene, which was observed in the presence of the $\beta-C D$, to the formation of an inclusion complex. Also, Coutouli-Argyropoulou et al. [20] reported on the electrochemical properties of derivatives of ferrocencein the presence of $\beta-C D$. Again, similar shifts in the peak potential for the oxidation of ferrocene derivatives was observed, indicating the formation of the inclusion complex, while Yanez et al. [21] obtained similar current and potential data for nifedipine (NF) and nicardipine (NC) in the presence of neutral $\beta-C D$.

\section{Experimental}

\subsection{Chemicals}

Dopamine, DA, sulfonated $\beta$-cyclodextrin sodium salt and sulfonated $\alpha$-cyclodextrin sodium salt were purchased from Sigma-Aldrich. The degree of sulfation of the randomly substituted $\beta$-cyclodextrin was reported as $7-11 \mathrm{~mol}$ of sulfonated groups per mol of $\beta$-cyclodextrin $(\beta-C D)$. It should be noted that the exact molar mass of the sulfonated CD is unknown and in cases where the concentration was required, e.g., in relation to the Job's plots, the molecular mass was computed using an average value of $9 \mathrm{~mol}$ of sulfonate per mol of $\beta-C D$. All other reagents were of analytical grade, obtained from either Sigma-Aldrich or Riedel de-Haen and were used as received. A citrate-phosphate buffer, with a $\mathrm{pH}$ of 6.0 (formed by mixing $0.2 \mathrm{~mol} \mathrm{dm}^{-3} \mathrm{Na}_{2} \mathrm{HPO}_{4}$ and $0.1 \mathrm{~mol} \mathrm{dm}^{-3}$ $\mathrm{C}_{6} \mathrm{H}_{8} \mathrm{O}_{7}$ ), was used as the supporting electrolyte. All solutions were prepared in deionised water. All DA-containing solutions were prepared immediately before use and were de-oxygenated with nitrogen to avoid the oxidation of DA.

\subsection{Instrumentation}

Cyclic voltammetry and rotating disc voltammetry were carried out using a Solartron Model SI 1285 potentiostat. All measurements were performed at room temperature $\left(24 \pm 2{ }^{\circ} \mathrm{C}\right)$ in a standard three-electrode cell with a glassy carbon (GC) electrode as the working electrode, SCE as the reference electrode and a high surface area platinum wire as the counter electrode. The GC electrodes ( $4 \mathrm{~mm}$ in diameter) were encased into a larger insulating Teflon sheath and set in place using a non-conducting epoxy resin. Electrical contact was achieved using a copper wire. The electrodes were polished to a smooth surface finish using diamond pastes (Buehler MetaDiMonocrystalline Diamond suspension) on

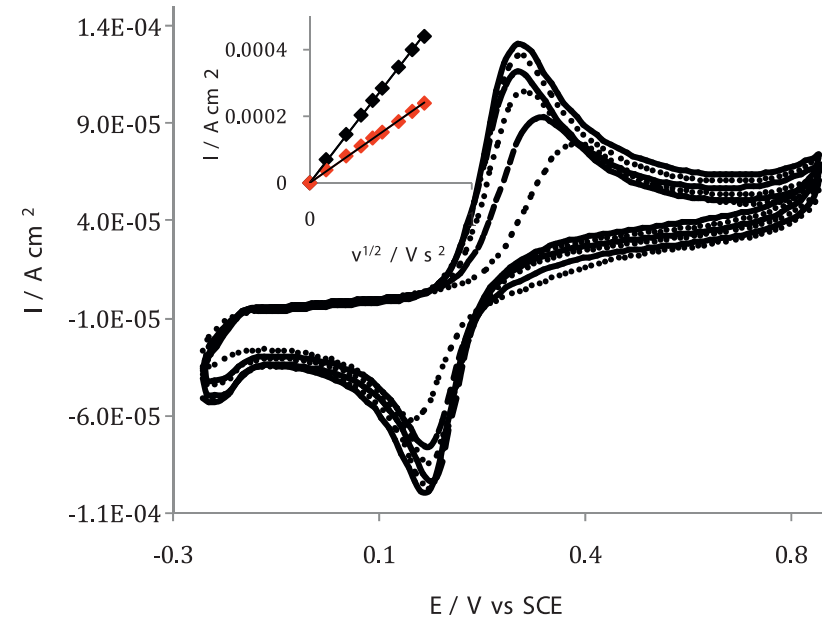

Fig. 2. Cyclic voltammograms recorded at a GC electrode at $50 \mathrm{mVs}^{-1}$ in $5.0 \times 10^{-4} \mathrm{~mol} \mathrm{dm}^{-3} \mathrm{DA}$ in a citrate-phosphate buffer, $\mathrm{pH} 6.0$, in the absence and presence of increasing concentrations of sulfonated $\beta$-CD. Inset the plot of $I_{\mathrm{p}} \mathrm{Vs} . v^{1 / 2}$ on GC disc electrode in citrate-phosphate buffer, $\mathrm{pH} 6.0$, for $5.0 \times 10^{-4} \mathrm{~mol} \mathrm{dm}^{-3} \mathrm{DA}$ in the absence and presence of $2.0 \times 10^{-2} \mathrm{~mol} \mathrm{dm}^{-3} \mathrm{~S} \beta-\mathrm{CD}$.

a Buehler micro-cloth, rinsed with water, sonicated to remove any polishing residues, rinsed again in water and finally dried.

The cyclic voltammetry experiments were recorded at $50 \mathrm{mV} \mathrm{s}^{-1}$ in the potential interval of -250 to $800 \mathrm{mV}$ vs. SCE. The DA concentration was maintained fixed at $5.0 \times 10^{-4} \mathrm{~mol} \mathrm{dm}^{-3}$ in the supporting electrolyte, while the concentration of the sulfonated $\beta$-CD host was varied to give solutions with an excess of the sulfonated $\beta-C D$. The steady-state rotating disc voltammograms were recorded in a similar electrochemical window with rotation speeds from 200 to $2000 \mathrm{rpm}$.

${ }^{1} \mathrm{H}-\mathrm{NMR}$ experiments were performed on a Bruker $300 \mathrm{MHz}$ NMR spectrometer at $293 \mathrm{~K}$ in $\mathrm{D}_{2} \mathrm{O}(>99.92 \%$ isotopic purity and purchased from Apollo Scientific). ${ }^{1} \mathrm{H}$ NMR peak protons were reported in ppm relative to the internal reference, tetramethylsilane $(\delta=0.0 \mathrm{ppm})$. A $0.1 \mathrm{~mol} \mathrm{dm}^{-3} \mathrm{KCl}$ was used to buffer the ionic strength, as the sulfonated $\beta-C D$ is highly charged and ionised. Varying amounts of sulfonated $\beta-C D$ dissolved in $0.1 \mathrm{~mol} \mathrm{dm}^{-3}$ $\mathrm{KCl} / \mathrm{D}_{2} \mathrm{O}$ were added to $0.5 \mathrm{~mL}$ volumes of a $5.0 \times 10^{-4} \mathrm{~mol} \mathrm{dm}^{-3}$ DA stock solution made up in $0.1 \mathrm{~mol} \mathrm{dm}^{-3} \mathrm{KCl} / \mathrm{D}_{2} \mathrm{O}$, to generate final sulfonated $\beta-C D$ concentrations that were in excess. The samples were allowed to equilibrate for $60 \mathrm{~min}$ before acquiring the ${ }^{1} \mathrm{H}$ NMR spectra. The aromatic region of the DAguest molecule was monitored to follow the formation of the inclusion complex, as the randomly substituted sulfonated $\beta$-CD had a complex ${ }^{1} \mathrm{H}$ NMR spectrum, making it too difficult to follow the resonance shifts of the protons from the sulfonated $\beta-C D$.

\section{Results and discussion}

\subsection{Evidence for the formation of an inclusion complex}

The influence of the sulfonated $\beta-C D$ on the cyclic voltammograms of DA can be seen in Fig. 2. Here, voltammograms are presented for increasing concentrations of sulfonated $\beta-C D$, but with a fixed $5.0 \times 10^{-4} \mathrm{~mol} \mathrm{dm}^{-3}$ concentration of DA. In the absence of the sulfonated $\beta-C D$, oxidation of DA is observed with a peak potential, $E_{\mathrm{p}}{ }^{\mathrm{A}}$, at $300 \mathrm{mV}$ vs. SCE, which corresponds to the oxidation of the DA to the o-dopamine-quinone. Upon reversal of the potential, a cathodic peak, $E_{\mathrm{p}}{ }^{\mathrm{C}}$, at $120 \mathrm{mV}$ vs. SCE is observed, corresponding to the reduction of o-dopamine-quinone back to DA. This is consistent with the well-known quasi-reversible electrochemistry of DA [13]. 


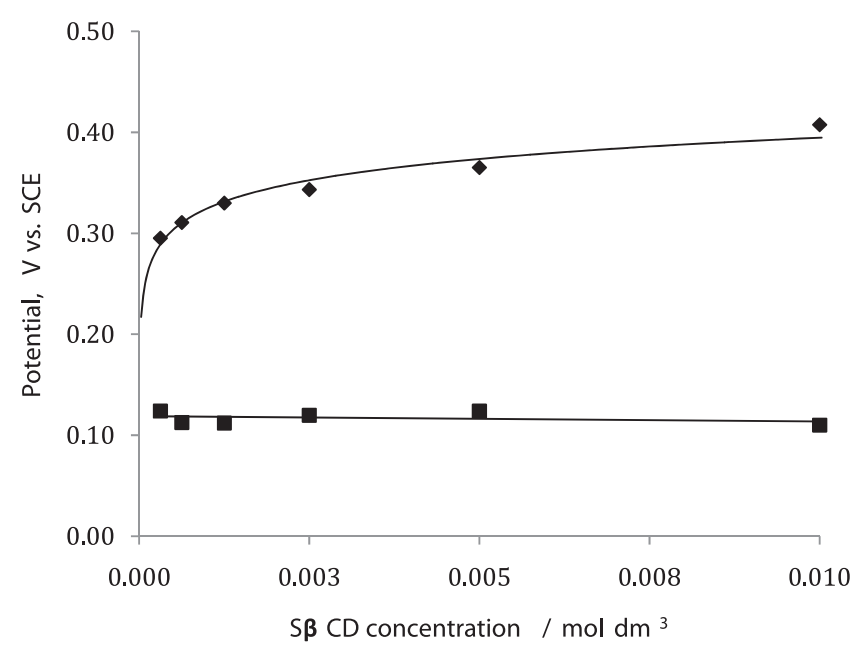

Fig. 3. The anodic $(\checkmark) E_{\mathrm{P}}{ }^{\mathrm{A}}$, and cathodic $(\mathbf{\square}), E_{\mathrm{P}}{ }^{\mathrm{C}}$, peak potentials recorded in a $5.0 \times 10^{-4} \mathrm{~mol} \mathrm{dm}^{-3}$ DA solution in a pH 6.0 citrate-phosphate buffer plotted as a function of the concentration of the sulfonated $\beta-C D$ in solution.

Although the voltammograms recorded in the presence of the sulfonated $\beta-C D$ have similar characteristics to that recorded in a pure DA solution, there are two significant differences. Firstly, there is a considerable decrease in the peak oxidation current, with increasing concentrations of sulfonated $\beta-C D$, which can be attributed to a decrease in the diffusion coefficient of DA. As shown in the inset in Fig. 2, there is a linear relationship between the peak current for the oxidation of DA and the square root of the scan rate, indicating that the reaction is under diffusion control, in agreement with the Randles-Sevcik equation. However, there is a significant difference in the slopes of the linear plots indicating a lower diffusion coefficient for the DA when in the presence of a large excess of the sulfonated $\beta-C D$. Secondly, there is a gradual increase in the peak oxidation potential with increasing concentrations of sulfonated $\beta-C D$. This indicates that it becomes more difficult to oxidize the DA molecule in the presence of the sulfonated $\beta-C D$. The shifts in the oxidation peak potentials are clearly evident in Fig. 3 , where the peak potentials for the oxidation of DA, $E_{\mathrm{p}}{ }^{\mathrm{A}}$, are shown as a function of the concentration of the sulfonated $\beta-C D$. A clear increase in $E_{\mathrm{p}}{ }^{A}$ is observed, varying from $300 \mathrm{mV}$ vs. SCE in the absence of the sulfonated $\beta-C D$ to $410 \mathrm{mV}$ vs. SCE in the presence of an excess of the sulfonated $\beta-C D$. However, there are no changes in the peak reduction potentials, $E_{\mathrm{p}}{ }^{\mathrm{C}}$; the $E_{\mathrm{p}}{ }^{\mathrm{C}}$ remains essentially constant at $120 \mathrm{mV}$ vs. SCE regardless of the concentration of the sulfonated $\beta-C D$.

There are two possible explanations that could account for these observations. Firstly, these data are consistent with the formation of an inclusion complex between the DA and the sulfonated $\beta$ $\mathrm{CD}$. Similar changes in the peak currents and potentials have been reported previously with other host-guest systems $[22,23]$. In particular, Dang et al. [22] reported similar results for the complexation of benzoquinone and anthraquinone with neutral $\beta-\mathrm{CD}$. A similar trend was observed by Gao et al. [23] in studying the complexation of basic brown $G$ with $\beta-C D$. However, the near constant reduction potentials, $E_{\mathrm{P}}{ }^{C}$, Fig. 3, indicate that the o-dopamine-quinone is expelled from the cavity, or that the binding constant for the o-dopamine-quinone-sulfonated $\beta$-CD inclusion complex is much lower than the inclusion complex formed with DA as the guest. Secondly, electrostatic interactions between the ionized sulfonated groups and the protonated DA molecule are possible and if these are sufficiently strong, a similar reduction in the diffusion coefficient of DA may be observed together with shifts in the peak potentials. However, the lack of any significant influence of the sulfonated
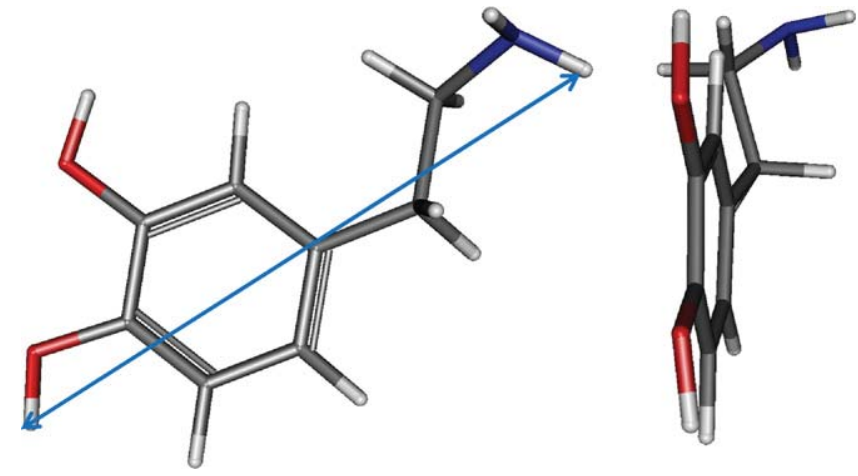

Fig. 4. Illustration of the DA molecule, its orientation and intermolecular distances from the furthest points.

$\beta-C D$ on the reduction of the protonated o-dopamine-quinone to DA suggests that a simple electrostatic interaction between the protonated amine group and the anionic sulfonated groups is not the dominant interaction.

To gain insight into the extent of these electrostatic interactions, similar experiments were carried out with the sulfonated $\alpha-C D$ and the neutral $\beta-C D$. The diameter of the $\alpha$-CD is smaller $(4.9 \AA)$ than the $\beta$-CD cavity $(6.2 \AA)$, as it only contains 6 glucose rings, but it has a similar degree of sulfation as the sulfonated $\beta-C D$, while the neutral $\beta-C D$ provides the larger sized cavity free from any anionic charge. The dimensions of the DA molecule are highlighted in Fig. 4. These data were obtained using a density functional theory (DFT) calculation. Illustrations of both the face and side geometry of the DA molecule are shown. The total diameter is taken from the $\mathrm{O}-\mathrm{H}$ group on the aromatic ring to the hydrogen on the nitrogen atom to give a total diameter of $8.95 \AA$, while the width of the aromatic ring, with the hydroxyl groups included, is $6.05 \AA$. The bulk of the molecule is planar. It is very clear based on these dimensions that DA cannot form an inclusion complex or fit within the cavity of the smaller sulfonated $\alpha-C D$.

The cyclic voltammograms recorded in a solution of $5.00 \times 10^{-4} \mathrm{~mol} \mathrm{dm}^{-3}$ DA were identical to those recorded with an excess of the sulfonated $\alpha-C D$, or an excess of the neutral $\beta-C D$. The anodic peak potentials and peak currents of DA in the absence and presence of a large excess of the CDs were identical. These results are very different to those presented in Fig. 2, and they provide clear evidence that the changes in the oxidation of DA in the presence of the sulfonated $\beta-C D$ are not simply due to electrostatic interactions between the protonated DA molecule and the anionic sulfonated groups on the $\beta-C D$. However, given the data recorded with the neutral $\beta-\mathrm{CD}$, it is clear that the anionic rim of the cyclodextrin facilitates the formation of an inclusion complex with the protonated DA molecule.

\subsection{Stoichiometry and structure of the inclusion complex}

The cyclic voltammetry data provide evidence that an inclusion complex is formed between the DA molecule and the sulfonated $\beta$ $\mathrm{CD}$ in solution. In order to obtain information on the stoichiometry of the inclusion complex, a Job's plot was generated. In this case, cyclic voltammograms were recorded in different DA-sulfonated $\beta$-CD solutions, where the mole fraction of DA was varied from 0.0 to 1.0 , in increments of 0.1 . The Job's plot was generated by following the changes in the peak oxidation current of DA using the relationship given in Eq. (1),

$\Delta i_{\mathrm{p}}=i_{\mathrm{po}}-i_{\mathrm{px}}$ 


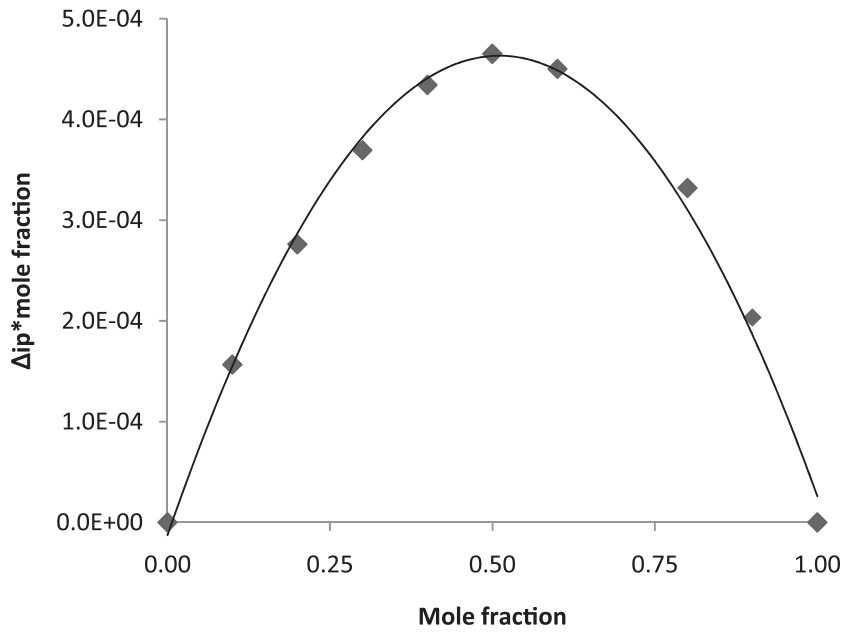

Fig. 5. Job's plot showing the difference in the peak oxidation current of DA, $\Delta i_{\mathrm{p}}$, as a function of the mole fraction of DA in a buffered sulfonated $\beta-C D$ solution.

where, $i_{\mathrm{po}}$ and $i_{\mathrm{px}}$ are the peak currents of DA in the absence and presence of the sulfonated $\beta-C D$, respectively. These $\Delta i_{\mathrm{p}}$ values were then multiplied by the corresponding mole fraction and the product was plotted as a function of the mole fraction. A typical plot is presented in Fig. 5. A clear maximum value is seen at a mole fraction of 0.5 , which confirms that the DA and sulfonated $\beta-C D$ bind and form an inclusion complex in a 1:1 stoichiometric ratio, i.e., one DA molecule is included in a single sulfonated $\beta$-CD cavity.

Further information on the nature of the inclusion complex was obtained using NMR spectroscopy. The chemical structure of DA, with the hydrogen atoms labeled as, a-H, b-H, c-H, d-H and e-H, is shown in Fig. 6(a). The protons designated as a-H, b-H and $\mathrm{c}-\mathrm{H}$ correspond to the aromatic protons while the protons, $\mathrm{d}-\mathrm{H}$ and $\mathrm{e}-\mathrm{H}$, correspond to the methylene $\left(-\mathrm{CH}_{2}-\right)$ group protons. Fig. 6(b) shows the aromatic region of the ${ }^{1} \mathrm{H}$ NMR spectra of $0.02 \mathrm{~mol} \mathrm{dm}^{-3}$ sulfonated $\beta-\mathrm{CD}, 5.0 \times 10^{-3} \mathrm{~mol} \mathrm{dm}^{-3} \mathrm{DA}$ and a mixture of DA and the sulfonated $\beta-C D$. The letters shown on the plot represent the aromatic protons depicted in Fig. 6(a), and serve to illustrate the chemical shift of the individual protons in the presence of the sulfonated $\beta-\mathrm{CD}$. On comparing the ${ }^{1} \mathrm{H}$ NMR spectrum of DA in the absence and presence of the sulfonated $\beta-C D$, the protons involved in the inclusion process can be identified. It is evident from Fig. 6(b) that the chemical shift of the a-H proton in DA is significant, with $\Delta \delta$ at $0.106 \mathrm{ppm}$, while the chemical shift of the $\mathrm{c}-\mathrm{H}$ is negligible, with $\Delta \delta$ at $0.002 \mathrm{ppm}$, indicating that it remains outside the $\mathrm{CD}$ cavity. This upfield, or low frequency shift, of the aromatic a-H protons on the DA molecule indicates a shielding effect, which is probably due to the increase in the electron density inside the cavity from the non-bonding electron pairs of the glycosidic oxygen bridges [24]. This is clear evidence that the aromatic ring of the DA molecule penetrates the cavity of the sulfonated $\beta-C D$. A schematic of the inclusion complex is presented in Fig. 6(c), which shows the c-H proton and the protonated amine group outside the cavity and the a-H proton deep within the cavity. It is highly probable that the protonated amine group is bound electrostatically by the anionic sulfonated groups on the rim of the cavity. Indeed, Bratu et al. [25] observed that the methylene groups of fenbufen remained outside the cavity of neutral $\beta-C D$ and the fenbufen molecule entered from the larger side or the secondary opening of the $\beta-C D$ ring. Similarly, Chao et al. [26] showed using NMR measurements that the aromatic ring of caffeic acid, a molecule with similarities to DA, lay inside the $\beta-C D$ cavity, while the more polar groups remained outside the cavity.
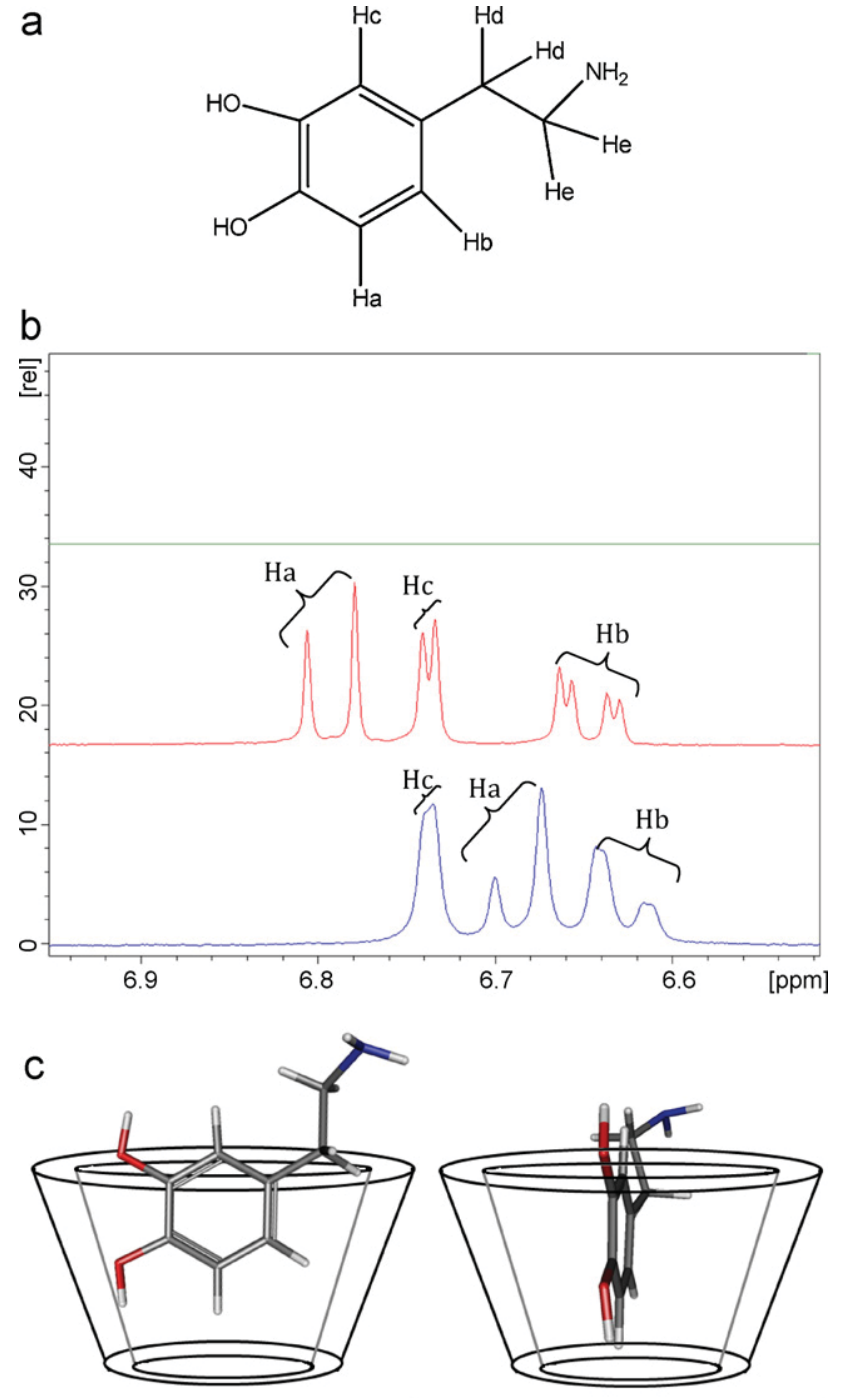

Fig. 6. (a) Labeled DA molecule, (b) ${ }^{1} \mathrm{H}$ NMR spectra recorded in $\mathrm{D}_{2} \mathrm{O}$ and $0.1 \mathrm{~mol} \mathrm{dm}^{-3} \mathrm{KCl}$ for (i) sulfonated $\beta-\mathrm{CD}$, (ii) $5 \times 10^{-4} \mathrm{~mol} \mathrm{dm}^{-3} \mathrm{DA}$, (iii) $5 \times 10^{-4} \mathrm{~mol} \mathrm{dm}^{-3} \mathrm{DA}$ with an excess of the sulfonated $\beta-\mathrm{CD}$. The corresponding protons are those designated in (a), (c) a schematic representation of the inclusion complex. Rotating-disc voltammograms of $5.0 \times 10^{-4} \mathrm{~mol} \mathrm{dm}^{-3} \mathrm{DA}$ recorded at $5 \mathrm{mV} \mathrm{s}^{-1}$ and $500 \mathrm{rpm}$ in a citrate-phosphate buffer in the (1) absence and presence of (2) $0.005 \mathrm{~mol} \mathrm{dm}^{-3}$ (3) $0.010 \mathrm{~mol} \mathrm{dm}^{-3}$ and (4) $0.020 \mathrm{~mol} \mathrm{dm}^{-3}$ sulfonated $\beta-C D$. The inset shows a plot of $E_{1 / 2}$ vs. the concentration of the sulfonated $\beta-C D$.

\subsection{Determination of $K$ and $k_{D A}$ in the presence of the sulfonated $\beta-C D$}

In order to calculate the association constant, $K$, a series of rotating disc voltammetry experiments was carried out. In Fig. 7 rotating disc voltammograms recorded at $500 \mathrm{rpm}$ are shown for the oxidation of DA in the absence and presence of increasing concentrations of sulfonated $\beta-C D$. There is a clear increase in the half-wave potentials, $E_{1 / 2}$, which is shown more clearly in the inset and a considerable reduction in the limiting current, $i_{\mathrm{L}}$, as the concentration of the sulfonated $\beta-C D$ is increased. Similar results were obtained at other rotation speeds, ranging from 200 to $2000 \mathrm{rpm}$. These data are in good agreement with the cyclic voltammograms presented in Fig. 2, and are consistent, again, with the formation of an inclusion complex. The diffusion coefficients of DA in the absence, $D_{f}$, and in the presence of an excess of the sulfonated $\beta-C D$, $D_{c}$, were obtained using Levich plots. A representative Levich plot is depicted in Fig. 8, while the variations in the diffusion coefficient 


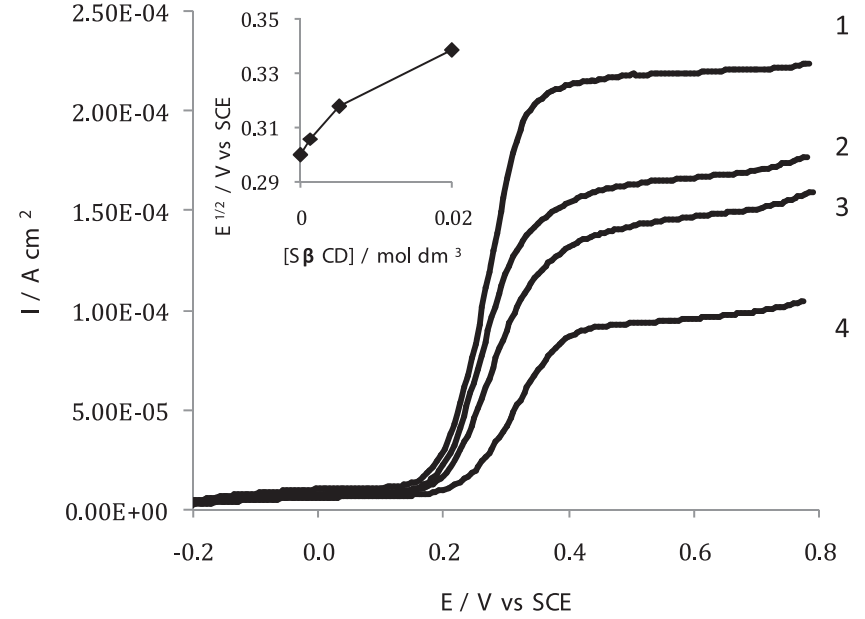

Fig. 7. Plot of $i_{\mathrm{L}}$ as a function of $\omega^{1 / 2}$ obtained from rotating-disc voltammograms recorded in $5.0 \times 10^{-4} \mathrm{~mol} \mathrm{dm}^{-3} \mathrm{DA}$ in a citrate-phosphate buffer in the (1) absence and presence of (2) $1.25 \times 10^{-3}$ (3) $2.50 \times 10^{-3}$ (4) $5.00 \times 10^{-3}$ (5) $1.00 \times 10^{-2}$ and (6) $2.00 \times 10^{-2} \mathrm{~mol} \mathrm{dm}^{-3}$ sulfonated $\beta$-CD. Inset shows the diffusion coefficients of DA plotted as a function of concentration of sulfonated $\beta-C D$.

of DA in the presence of increasing concentrations of sulfonated $\beta$ $\mathrm{CD}$ are shown in the inset in the figure. These diffusion coefficients were obtained using the Levich relationship, Eq. (2), where $i_{\mathrm{L}}$ is the limiting current density, $n$ is the number of electrons transferred, $F$ is Faraday's constant, $D$ is the diffusion coefficient, $\mathrm{cm}^{2} \mathrm{~s}^{-1}, v$ is the kinematic viscosity (a value of $0.92 \times 10^{-6} \mathrm{~m}^{2} \mathrm{~s}^{-1}$ was used for the system), $c$ is the concentration and $\omega$ is the rotational speed in $\operatorname{rad~} \mathrm{s}^{-1}$.

$i_{\mathrm{L}}=0.621 n F D^{2 / 3} v^{-1 / 6} c \omega^{1 / 2}$

A clear decrease in the diffusion coefficient was found with increasing concentration of sulfonated $\beta-\mathrm{CD}$. For example, a $D_{\mathrm{f}}$ value of $5.11 \times 10^{-6} \mathrm{~cm}^{2} \mathrm{~s}^{-1}$ was found for free DA, while the diffusion coefficient of the complexed species, $D_{\mathrm{c}}$, was evaluated as $2.44 \times 10^{-6} \mathrm{~cm}^{2} \mathrm{~s}^{-1}$. This latter value was computed with a large excess of the cyclodextrin, i.e., approximately $0.2 \mathrm{~mol} \mathrm{dm}^{-3}$ sulfonated $\beta$-CD with $1.0 \times 10^{-4} \mathrm{~mol} \mathrm{dm}^{-3} \mathrm{DA}$, to drive the equilibrium to favour the complexed DA species. These diffusion coefficients for DA are in close agreement with values reported in the literature [27,28], which vary between $1.9 \times 10^{-6}$ and $6.3 \times 10^{-6} \mathrm{~cm}^{2} \mathrm{~s}^{-1}$. Again, the decrease in the diffusion coefficient of DA can be attributed to the formation of a DA-sulfonated $\beta-C D$ inclusion complex, which has a slower diffusion coefficient due to the large size of the cyclodextrin. This gives a $D_{\mathrm{c}} / D_{\mathrm{f}}$ ratio of 0.47 , which is in very good agreement with ratios found in the literature for the complexation of ferrocenes with $\beta$-CDs $[20,29]$. Radi and Eissa [5] also observed a change in the diffusion coeffiecent of Indapamide in the presence and absence of $\beta-C D$ similar to what is shown here.

Using Eq. (3), the association constant, $K$, was computed as $331.28 \pm 5.85$. In this analysis, $T$ is the thermodynamic temperature, $\left(E_{1 / 2}\right)_{\text {app }}$ and $\left(E_{1 / 2}\right)_{\mathrm{f}}$ are the half-wave potentials of the electroactive guest obtained in the presence and absence of the sulfonated $\beta-C D$, respectively.

$$
\left(\frac{F}{R T}\right)\left\{\left(E_{1 / 2}\right)_{\mathrm{app}}-\left(E_{1 / 2}\right)_{\mathrm{f}}\right\}=\ln (1+K[S \beta-C D])+\ln \left(\frac{D_{\mathrm{c}}}{D_{\mathrm{f}}}\right)^{1 / 2}
$$

Additionally, the Koutecky-Levich equation, Eq. (4), was used to calculate the heterogeneous charge transfer rate constant, $k_{\mathrm{DA}}$, for DA in the absence and presence of the sulfonated $\beta-C D$. In this analysis, $i_{\mathrm{L}}$ represents the measured limiting current, $i_{K}$ is the current of the electron transfer between the DA and the electrode, $i_{\text {lev }}$ is the Levich current, which corresponds to the mass transfer of DA in the solution, $k_{\mathrm{DA}}$ is the reaction rate constant and is the surface coverage.

$\frac{1}{i_{\mathrm{L}}}=\frac{1}{i_{\mathrm{K}}}+\frac{1}{i_{\mathrm{lev}}}=\frac{1}{n F A \Gamma k_{\mathrm{DA}} \mathrm{C}}+\frac{1.61}{n F A v^{-1 / 6} D^{2 / 3} \omega^{1 / 2} \mathrm{C}}$

Fig. 9 shows the Koutecky-Levich plots for DA alone and in the presence of varying concentrations of sulfonated $\beta-C D$. For each sulfonated $\beta-C D$ concentration, a linear Koutecky-Levich relationship was observed and from the $y$-intercept of each plot the $k$ values were obtained. These are shown as a function of the concentration of the sulfonated $\beta-C D$ in the inset in the figure. The $k$ value was computed as $1.26 \times 10^{-2} \mathrm{~cm} \mathrm{~s}^{-1}$ for DA in the absence of the

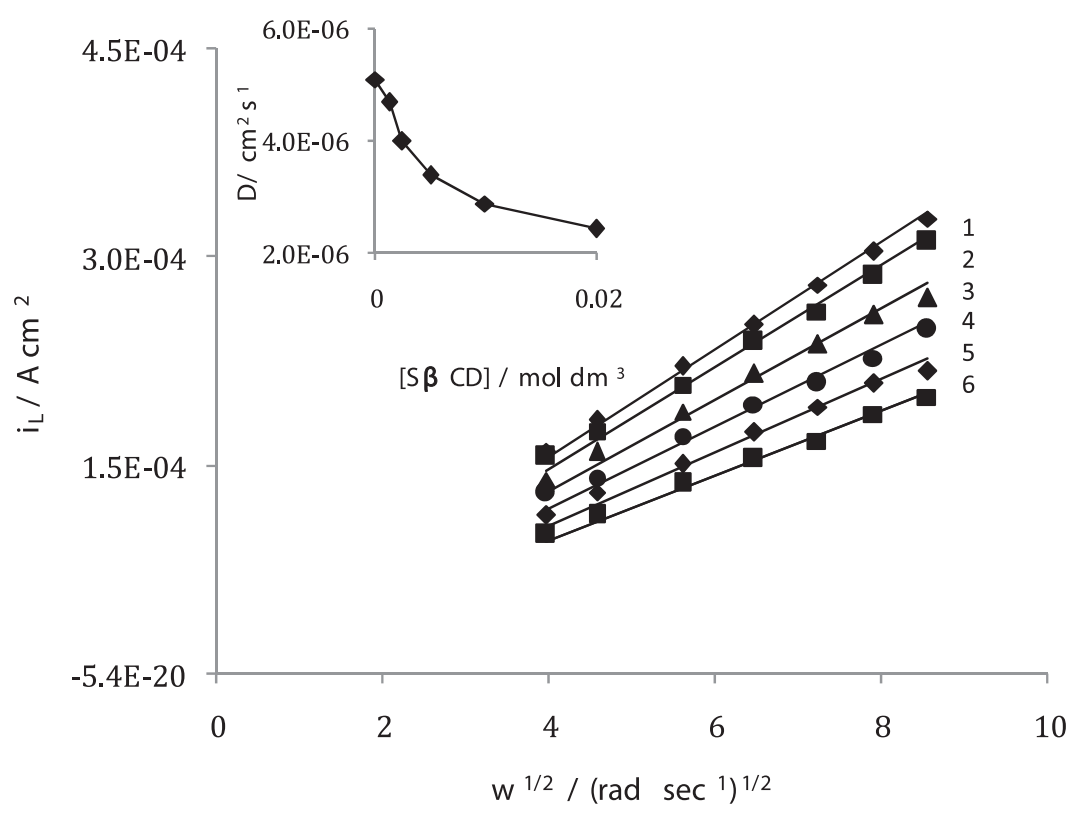

Fig. 8. Plot of $i_{\mathrm{L}}$ versus $\omega^{1 / 2}$ at rotating-disc voltammograms of DA $\left(5.0 \times 10^{-4} \mathrm{~mol} \mathrm{dm}^{-3}\right)$ on a GC disc electrode in citrate-phosphate buffer, (pH=6), in the presence of varying amounts of $[\mathrm{S} \beta-\mathrm{CD}] / \mathrm{mol} \mathrm{dm} \mathrm{m}^{-3}$. (1) 0.00 (2) $1.25 \times 10^{-3}$ (3) $2.50 \times 10^{-3}$ (4) $5.00 \times 10^{-3}$ (5) $1.00 \times 10^{-2}$ and (6) $2.00 \times 10^{-2}$. The inset shows a plot of the diffusion coefficients as a function of increasing $\mathrm{S} \beta-\mathrm{CD}$ concentrations. 


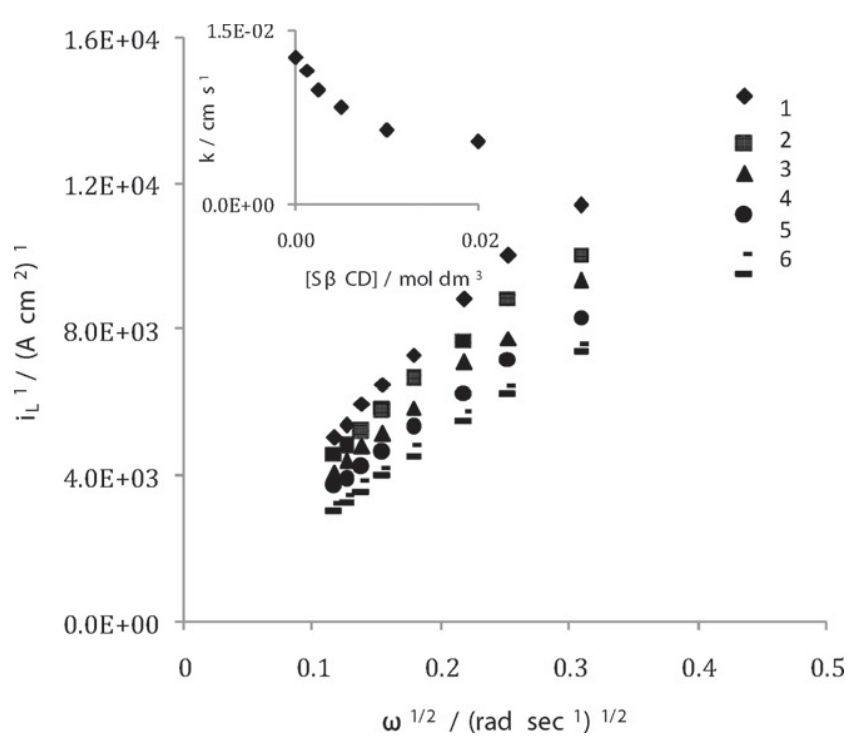

Fig. 9. Koutecky-Levich plots for DA in the absence and presence of varying concentrations of $[S \beta-C D] / \mathrm{mol} \mathrm{dm}^{-3}$. (1) $2.00 \times 10^{-2}$ (2) $1.00 \times 10^{-2}$ (3) $5.00 \times 10^{-3}$ (4) $2.50 \times 10^{-3}(5) 1.25 \times 10^{-3}$ and (6) 0.00 . Experiments were carried out in a citratephosphate buffer $(\mathrm{pH}=6.0)$. Current was read at $+0.700 \mathrm{~V}$. The inset shows the rate constant as a function of increasing $S \beta-C D$ concentrations.

sulfonated $\beta$-CD and $5.37 \times 10^{-3} \mathrm{~cm} \mathrm{~s}^{-1}$ in the presence of a large excess of the sulfonated $\beta-C D$. Typical rate constants for the oxidation of DA in the literature are in the range of $1.3 \times 10^{-2} \mathrm{~cm} \mathrm{~s}^{-1}$ to $3.88 \times 10^{-3} \mathrm{~cm} \mathrm{~s}^{-1}$ on different materials and the higher values are in good agreement with the values obtained here [30,31]. It is clearly evident that the rate constant for the oxidation of DA decreases as the concentration of the sulfonated $\beta-C D$ is increased. This decrease in the rate constant is consistent with the formation of an inclusion complex. It is reasonable to expect that the rate of the electron-transfer reaction will be higher when the DA is free in solution, compared to when it is complexed with in the cavity of the sulfonated $\beta-C D$. Using Marcus theory this can be explained in terms of the reorganization energy. The re-organization energy is likely to be higher when the DA is confined within the cavity of the sulfonated $\beta-C D$, especially if the ortho-quinone is expelled from the cavity once it is formed, as indicated in Fig. 3. These events will contribute to a higher reorganization energy leading to a lower rate of reaction.

\section{Conclusions}

DA forms a weak inclusion complex with sulfonated $\beta-C D$ in aqueous solution. Clear evidence for the formation of the inclusion complex was obtained from cyclic voltammetry, rotating disc voltammetry and NMR spectroscopy measurements. The inclusion complex was formed as a 1:1 stoichiometry of DA to the sulfonated $\beta-C D$, while NMR analyses showed that the aromatic ring of the DA molecule resides within the cyclodextrin cavity. The protonated amine group remains outside the cavity, bound electrostatically with the anionic sulfonated groups. The size of the cavity plays an important role, as no inclusion complex was formed between DA and the sulfonated $\alpha-\mathrm{CD}$. The association constant, $K_{\mathrm{f}}$, was computed as $331.3 \pm 5.8$ using rotating disc voltammetry, indicating a weak inclusion complex. The rate constant for the oxidation of DA was found to decrease on formation of the inclusion complex. This was attributed to larger reorganization energy for the oxidation of the included DA.

\section{Acknowledgements}

The authors would like to acknowledge the Irish Research Council for Science and Technology (IRCSET) and the John and Pat Hume Scholarship programme at the National University of Ireland Maynooth, Ireland for funding this work.

\section{References}

[1] J. Szejtli, Chem. Rev. 98 (1998) 1743.

[2] A. Harada, Y. Takashima, H. Yamaguchi, Chem. Soc. Rev. 38 (2009) 875

[3] A.R. Hedges, Chem. Rev. 98 (1998) 2035.

[4] L. Liu, Q.X. Guo, J. Incl. Phenom. Macro. 42 (2002) 1.

[5] A.E. Radi, S. Eissa, J. Incl. Phenom. Macro. 71 (2011) 95.

[6] I.V. Terekhova, M. Kozbial, R.S. Kumeev, G.A. Alper, J. Solution Chem. 40 (2011) 1435.

[7] M. Vilar, M. Navarro, Electrochim. Acta 56 (2011) 305.

[8] G. Wenz, Angew. Chem. Int. Ed. Engl. 33 (1994) 803.

[9] V. Zia, R.A. Rajewski, V.J. Stella, Pharm. Res. 18 (2001) 667.

[10] A. Amini, T. Rundlof, M.B.G. Rydberg, T. Arvidsson, J. Sep. Sci. 27 (2004) 1102.

[11] F.T.A. Chen, G. Shen, R.A. Evangelista, J. Chromatogr. A 924 (2001) 523.

[12] G.K.E. Scriba, J. Sep. Sci. 31 (2008) 1991.

[13] T. Luczak, Electroanalysis 20 (2008) 1639.

[14] S. Gadde, A.E. Kaifer, Curr. Org. Chem. 15 (2011) 27.

[15] A. Garg, B. Gupta, R. Prakash, S. Singh, Chem. Pharm. Bull. 58 (2010) 1313.

[16] A.E. Radi, S. Eissa, Electroanalysis 22 (2010) 2991.

[17] K. Srinivasan, J. Vaheethabanu, P. Manisankar, T. Stalin, J. Mol. Struct. 987 (2011) 214.

[18] C. Yanez, M. Araya, S. Bollo, J. Incl. Phenom. Macro. 68 (2010) 237.

[19] V.V. Strelets, I.A. Mamedjarova, M.N. Nefedova, N.I. Pysnograeva, V.I. Sokolov, L. Pospisil, J. Hanzlik, J. Electroanal. Chem. 310 (1991) 179.

[20] E. Coutouli-Argyropoulou, A. Kelaidopoulou, C. Sideris, G. Kokkinidis, J. Electroanal. Chem. 477 (1999) 130.

[21] C. Yanez, L.J. Nunez-Vergara, J.A. Squella, Electroanalysis 15 (2003) 1771

[22] X.J. Dang, M.Y. Nie, J. Tong, H.L. Li, J. Electroanal. Chem. 448 (1998) 61.

[23] Z.N. Gao, X.L. Wen, H.L. Li, Pol. J. Chem. 76 (2002) 1001.

[24] H. Dodzuik, Cyclodextrins and Their Complexes, Wiley-VCH, 2006

[25] I. Bratu, J.M. Gavira-Vallejo, A. Hernanz, M. Bogdan, G. Bora, Biopolymers 73 (2004) 451.

[26] J.B. Chao, H.B. Tong, Y.F. Li, L.W. Zhang, B.T. Zhang, Supramol. Chem. 20 (2008) 461.

[27] D.W.M. Arrigan, M. Ghita, V. Beni, Chem. Commun. (2004) 732.

[28] V.S. Vasantha, S.M. Chen, J. Electroanal. Chem. 592 (2006) 77.

[29] T. Matsue, D.H. Evans, T. Osa, N. Kobayashi, J. Am. Chem. Soc. 107 (1985) 3411.

[30] F.N. Rein, R.C. Rocha, H.E. Toma, J. Inorg. Biochem. 85 (2001) 155.

[31] E. Winter, L. Codognoto, S. Rath, Anal. Lett. 40 (2007) 1197. 